\title{
Defect Control of Conventional and Anomalous Electron Transport at Complex Oxide Interfaces
}

\author{
F. Gunkel* \\ Institute for Electronic Materials, IWE2, RWTH Aachen University, 52074 Aachen, Germany; \\ Stanford Institute for Materials and Energy Sciences, SLAC National Accelerator Laboratory, \\ Menlo Park, California 94025, USA; \\ and Peter Gruenberg Institute, Forschungszentrum Juelich GmbH, \\ and Juelich Aachen Research Alliance for Fundamentals on Future Information Technology (JARA-FIT), \\ 52428 Juelich, Germany \\ Chris Bell \\ Stanford Institute for Materials and Energy Sciences, SLAC National Accelerator Laboratory, \\ Menlo Park, California 94025, USA; \\ and School of Physics, University of Bristol, Bristol BS8 1TL, United Kingdom \\ Hisashi Inoue, Bongju Kim, Adrian G. Swartz, and Tyler A. Merz \\ Geballe Laboratory for Advanced Materials, Department of Applied Physics, \\ Stanford University, Stanford, California 94305, USA \\ Yasuyuki Hikita \\ Stanford Institute for Materials and Energy Sciences, SLAC National Accelerator Laboratory, \\ Menlo Park, California 94025, USA \\ Satoshi Harashima \\ Stanford Institute for Materials and Energy Sciences, SLAC National Accelerator Laboratory, \\ Menlo Park, California 94025, USA; \\ and Department of Applied Physics, The University of Tokyo, \\ Bunkyo-ku, Tokyo 113-8654, Japan \\ Hiroki K. Sato \\ Stanford Institute for Materials and Energy Sciences, SLAC National Accelerator Laboratory, \\ Menlo Park, California 94025, USA; \\ and Department of Advanced Materials Science, The University of Tokyo, Chiba 277-8561, Japan \\ Makoto Minohara \\ Stanford Institute for Materials and Energy Sciences, SLAC National Accelerator Laboratory, \\ Menlo Park, California 94025, USA; \\ Geballe Laboratory for Advanced Materials, Department of Applied Physics, Stanford University, \\ Stanford, California 94305, USA; \\ and Photon Factory, High Energy Accelerator Research Organization (KEK), Tsukuba 305-0801, Japan \\ Susanne Hoffmann-Eifert and Regina Dittmann \\ Peter Gruenberg Institute, Forschungszentrum Juelich GmbH, and Juelich Aachen Research Alliance for \\ Fundamentals on Future Information Technology (JARA-FIT), 52428 Juelich, Germany \\ Harold Y. Hwang \\ Stanford Institute for Materials and Energy Sciences, SLAC National Accelerator Laboratory, \\ Menlo Park, California 94025, USA; \\ and Geballe Laboratory for Advanced Materials, Department of Applied Physics, Stanford University, \\ Stanford, California 94305, USA
}

(Received 28 December 2015; revised manuscript received 29 July 2016; published 30 August 2016)

*gunkel@iwe.rwth-aachen.de

Published by the American Physical Society under the terms of the Creative Commons Attribution 3.0 License. Further distribution of this work must maintain attribution to the author(s) and the published article's title, journal citation, and DOI. 
Using low-temperature electrical measurements, the interrelation between electron transport, magnetic properties, and ionic defect structure in complex oxide interface systems is investigated, focusing on $\mathrm{NdGaO}_{3} / \mathrm{SrTiO}_{3}(100)$ interfaces. Field-dependent Hall characteristics $(2-300 \mathrm{~K})$ are obtained for samples grown at various growth pressures. In addition to multiple electron transport, interfacial magnetism is tracked exploiting the anomalous Hall effect (AHE). These two properties both contribute to a nonlinearity in the field dependence of the Hall resistance, with multiple carrier conduction evident below $30 \mathrm{~K}$ and AHE at temperatures $\lesssim 10 \mathrm{~K}$. Considering these two sources of nonlinearity, we suggest a phenomenological model capturing the complex field dependence of the Hall characteristics in the low-temperature regime. Our model allows the extraction of the conventional transport parameters and a qualitative analysis of the magnetization. The electron mobility is found to decrease systematically with increasing growth pressure. This suggests dominant electron scattering by acceptor-type strontium vacancies incorporated during growth. The AHE scales with growth pressure. The most pronounced AHE is found at increased growth pressure and, thus, in the most defective, low-mobility samples, indicating a correlation between transport, magnetism, and cation defect concentration.

DOI: 10.1103/PhysRevX.6.031035

\section{INTRODUCTION}

The electron system emerging at interfaces between polar and nonpolar oxides [1-3] shows fascinating properties such as metallicity [1], superconductivity [4], strong electron correlations, and magnetic ordering [5-10]. The understanding of these properties and, in particular, the interrelation between ionic defects and electronic or magnetic properties is extensively debated. To this end, it has been shown that gradual ionic defect distributions can cause space charges and inhomogeneous electronic carrier concentrations at oxide interfaces [11-13].

For the prototypical interface system $\mathrm{LaAlO}_{3} / \mathrm{SrTiO}_{3}$ (LAO/STO), experimental and theoretical studies revealed a highly intricate band structure [14-17], orbital reconstructions and interactions accompanied with a similarly complex ionic defect structure [18-21]. These features give rise to highly complex and unusual behavior of the magnetoresistance and the Hall effect at low temperatures $[10,22,23]$. It is generally accepted that the polar discontinuity at these interfaces drives a mixed ionic-electronic interface reconstruction. However, it is still a matter of debate as to how the ionic defect structure varies with growth parameters and/or postdeposition annealing procedures and how the resultant ionic defect structure affects electron transport [19-21,24].

Another ongoing debate is the origin and nature of the interfacial magnetism in these systems. While some bulk $[6,8]$ and more surface-sensitive $[7,25]$ methods have observed signatures of magnetism without being able to identify the elemental origin, $\mathrm{x}$-ray magnetic circular dichroism has directly detected an in-plane magnetic moment on the Ti site in LAO/STO $[9,10]$. However, in other cases magnetism was not observed in LAO/STO $[26,27]$, suggesting that the magnetism may be highly sensitive to sample growth conditions. Indeed, the correlations between the electronic-ionic structure and the
Subject Areas: Condensed Matter Physics, Electronics, Magnetism

magnetic properties are still unclear. Theoretical studies have predicted both intrinsic and extrinsic origins for the magnetism at these oxide interfaces [28-31].

In this study, we focus on the electronic and magnetic properties of $\mathrm{NdGaO}_{3} / \mathrm{SrTiO}_{3}$ (NGO/STO) heterostructures. Sharing a similar ionic structure with LAO $\left(\mathrm{A}^{3+} \mathrm{B}^{3+} \mathrm{O}_{3}\right)$, NGO generally causes a similar interface reconstruction as LAO when grown on $\{100\}$ STO [32-34]. However, NGO possesses $\mathrm{Nd}^{3+}$ ions carrying a magnetic moment that is not present in LAO. (NGO is paramagnetic with an antiferromagnetic phase transition at about $1 \mathrm{~K}$ [35].) Hence, $\mathrm{NGO} / \mathrm{STO}$ is an interesting candidate system to induce, alter, or enhance interfacial magnetism in STO.

One route to indirectly access magnetism at the interface is by exploiting its influence on the magnetotransport due to the anomalous Hall effect (AHE) $[15,16,36,37]$. The contribution of the anomalous Hall effect is proportional to the magnetization component perpendicular to the current flow, $R_{x y}^{\mathrm{AHE}} \propto M_{z}$. The AHE is caused by scattering mechanisms other than the Lorentz force and has fundamentally different origins, such as intrinsic, skew scattering, and side jump mechanisms $[36,38,39]$. The total Hall resistance is therefore given by

$$
R_{x y}=R_{x y}^{\mathrm{NHE}}+R_{x y}^{\mathrm{AHE}} .
$$

The conventional Hall effect $R_{x y}^{\mathrm{NHE}}$ allows the extraction of the electron concentration and electron Hall mobility, yielding information about the defect structure of the sample. Therefore, a single transport measurement enables one to investigate (1) the ionic defect structure and (2) the magnetic properties of a specific NGO/STO interface. A comparison of these two properties in differently grown samples may yield a possible relation between the two.

In simple electronic systems, the field dependence of the AHE - and thus the field dependence of the magnetization 
$M_{z}$ - can be extracted directly from Hall data by subtraction of a linear normal Hall component [36,40]. However, in STO-based two-dimensional electron systems, the normal Hall effect itself can show a nonlinear field dependence $[10,16,41-43]$. Therefore, the direct extraction of the AHE requires the appropriate modeling of the entire field dependence of the Hall effect.

Here, we report on electronic transport measurements $(2-300 \mathrm{~K})$ at the interface of NGO/STO heterostructures grown at various oxygen pressures. For electrical characterization, we use a standard Hall bar geometry with the external field $B$ applied perpendicular to the NGO/STO interface. In order to account for a nonlinear behavior of the Hall resistance, we first apply a semiclassical model of multiple carrier conduction comprising two populations of electrons with concentrations $\left(n_{1}, n_{2}\right)$ and mobilities $\left(\mu_{1}, \mu_{2}\right)$, respectively, and discuss their dependence on growth pressure. We find that these transport parameters scale systematically with growth parameters providing important information about the ionic defect structure established during growth.

We then show that a correction term has to be introduced in order to account for an additional feature in the Hall coefficient arising below about $10 \mathrm{~K}$. This correction term is attributed to the AHE associated with magnetic ordering at the NGO/STO interface. We suggest a phenomenological model and apply it to the entire $B$-field dependence of the Hall coefficient. Within this model we are able to extract the AHE component and its field and temperature dependence. It is found that the critical temperature at which the AHE arises $T_{c}$, as well as its magnitude, can be controlled by the growth conditions during sample fabrication. The systematic trend found for samples grown at various oxygen partial pressure indicates a correlation between defect structure and AHE and, thus, an influence of defect structure on the magnetic properties of the NGO/STO interface. In particular, we reveal a trend that excludes oxygen vacancies as the origin of magnetism in these samples. Our analysis represents the first comprehensive modeling of the entire nonlinear behavior of the Hall effect in two-dimensional electron gases (2DEGs) at oxide interfaces, involving both multiple channel conduction and magnetism.

\section{SAMPLE PREPARATION}

We deposit eight unit cells thick NGO films on $\mathrm{TiO}_{2}{ }^{-}$ terminated $\{100\}$ STO substrates by pulsed laser deposition (PLD) at a temperature of $700^{\circ} \mathrm{C}$ and a laser fluence of $1.4 \mathrm{~J} / \mathrm{cm}^{2}$. The deposition pressure $p_{\text {dep }}$ is varied from $1 \times 10^{-2}$ to $4 \times 10^{-5}$ mbar oxygen. After deposition, the samples are cooled down at a constant rate of $10 \mathrm{~K} / \mathrm{min}$ at the deposition pressure. In this way, the $p \mathrm{O}_{2}$-dependent thermodynamic state varies from sample to sample $[18,20]$.

As reference samples, we grow LAO/STO $\left(700^{\circ} \mathrm{C}\right.$, $1.9 \mathrm{~J} / \mathrm{cm}^{2}, p_{\text {dep }}=1 \times 10^{-3} \mathrm{mbar}$ ) and $\mathrm{LaGaO}_{3}$ (LGO)/ STO $\left(700^{\circ} \mathrm{C}, 1.4 \mathrm{~J} / \mathrm{cm}^{2}, p_{\text {dep }}=4 \times 10^{-5} \mathrm{mbar}\right)$ samples.
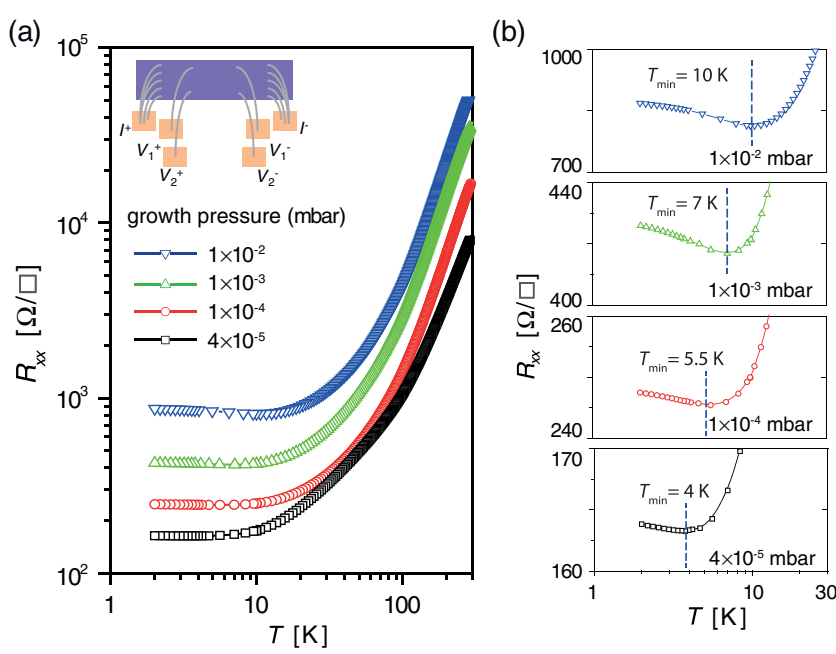

FIG. 1. (a) Sheet resistance $R_{x x}$ obtained at zero field ( $B=0 \mathrm{~T}$ ) for NGO/STO heterostructures grown at various $p_{\mathrm{dep}}$. (b) $R_{x x}$ in the low-temperature regime: A slight resistance upturn is observed for all samples, while the temperature of minimum resistance $T_{\min }$ scales with $p_{\mathrm{dep}}$.

For all deposition conditions and materials, reflection high-energy electron diffraction intensity oscillations are observed during growth, indicating a layer-by-layer growth mode (growth rate approximately $30-40 \mathrm{~s}$ per unit cell at a laser repetition rate of $1 \mathrm{~Hz}$ ). Atomic force microscopy shows that all films exhibit an atomically smooth surface morphology.

For the transport measurements, the samples are cut into $1 \times 5$ - $\mathrm{mm}^{2}$-sized bars. Contacts for current injection $\left(I^{+}, I^{-}\right)$are achieved by Al-wire bonding across the entire width of the sample to ensure a homogeneous current density. Contacts for $R_{x x}\left(V_{1}^{+}, V_{1}^{-}\right)$and $R_{x y}\left(V_{1}^{+}, V_{2}^{+}\right)$ measurements in four-point geometry are realized by single wire bonds. The bonding wires provide Ohmic contacts to the interface 2DEG at the edge of the bars, ensuring minimal impact on the current flow. Therefore, good comparability to lithographically obtained Hall bar structures can be assumed. For each measurement, we use a second channel to check homogeneity. The sample geometry is sketched in the inset of Fig. 1(a).

\section{GROWTH, THERMODYNAMICS, AND DEFECT STRUCTURE}

PLD growth involves complex, coupled processes so that the growth conditions can have a large impact on the resulting film and interface defect structure [44-48]. A variation of the growth pressure, in particular, can lead to altering oxidation states of the incoming plasma species as well as altering growth kinetics $[49,50]$.

The cation stoichiometry of complex oxide thin films (here, the $\mathrm{Nd} / \mathrm{Ga}$ ratio) primarily depends on the applied laser fluence $[44,47,48,51]$. However, for the particular 
case of NGO, another important process is evaporation of volatile $\mathrm{Ga}$ species from the film. This process is controlled via the growth temperature [32]. Oxidation of $\mathrm{Ga}$ enhances its thermal stability [52]. Therefore, we observe an increase of the Ga concentration in the NGO films with increasing growth pressure as revealed by $\mathrm{x}$-ray photoemission spectroscopy.

Although PLD growth is generally considered a nonequilibrium process, thermodynamic considerations also play an important role. During and after growth, the system strives for a defined equilibrium state involving both ionic and electronic reconfigurations. The growing heterostructure tends to approach as much as possible its equilibrium state that is controlled by oxygen pressure and temperature $[18,53,54]$. For $n$-type STO, thermodynamics imply a decreasing equilibrium concentration of oxygen vacancies $\mathrm{V}_{\mathrm{O}}^{*}$ with increasing ambient oxygen pressure (i.e., $p_{\text {dep }}$ ), and for an activated $\mathrm{Sr}$ sublattice, an increasing concentration of strontium vacancies, $\left[\mathrm{V}_{\mathrm{Sr}}^{\prime \prime}\right] \propto\left[\mathrm{V}_{\mathrm{O}}^{*}\right]^{-1}[53,55]$. In $n$-type STO, strontium vacancy defects are the dominant ionic defect species, increasingly incorporated upon oxidation, as extensively discussed in the literature [53,54,56-61]. Sr vacancies are typically induced via the partial Schottky equilibrium, which is equivalent to the formation of $\mathrm{SrO}$ in an oxidizing atmosphere leaving behind a vacant strontium lattice site [53,62]. In STO, the formation of strontium vacancies exceeds the formation of titanium vacancies, which are energetically and kinetically less favorable $[53,62,63]$. Note that cation diffusion in STO is much slower than anion (oxygen) diffusion. On short length scales close to surfaces, however, cation vacancy incorporation can be considerably fast $[56,61]$.

In the particular case of $n$-type oxide heterostructure interfaces, it has been found that-similar to bulk $n$-doped STO-Sr vacancies can be induced by thermodynamic equilibrium processes [20,21], as well as by postdeposition annealing, driving the interfacial Schottky disorder towards equilibrium [24]. As shown in Ref. [24], $\mathrm{Sr}$ vacancy incorporation has a significant impact on the low-temperature transport of LAO/STO interfaces, involving resistance increase and the emergence of a pronounced resistance upturn.

In summary, for the samples we investigate in this study, the processes discussed above should lead to a relative decrease in the number of oxygen vacancies in samples deposited at higher $p_{\text {dep }}$ (due to oxidation). In addition, growth kinetics suggest a reduced rate of intermixing of cations between the thin film and substrate at increased growth pressure [19]. Thermodynamic processes yield an increasing effect of disorder induced by intrinsic cation vacancies in STO $[20,24,53,55]$. As we show, this scenario is consistent with the corresponding transport data revealing a systematic decrease in electron mobility at increased growth pressure (Sec. IV B).

\section{RESULTS}

\section{A. Temperature dependence of Hall characteristics in $\mathrm{NdGaO}_{3} / \mathrm{SrTiO}_{3}$ heterostructures}

First, we investigate the temperature dependence of the sheet resistance of the NGO/STO interface $R_{x x}$ at zero field. For $T \gtrsim 10 \mathrm{~K}$, all measured NGO/STO samples show metallic behavior, as depicted in Fig. 1(a). Comparing the various samples, $R_{x x}$ shows a general trend over the entire investigated temperature range $(2-300 \mathrm{~K})$ : The highest resistance is observed for the NGO/STO heterostructure grown at the highest deposition pressure, $p_{\text {dep }}=1 \times 10^{-2}$ mbar, while the resistance characteristics systematically shift towards lower $R_{x x}$ values when decreasing $p_{\text {dep }}$. The residual resistance at low temperatures $(\lesssim 10 \mathrm{~K})$ ranges from $160 \Omega / \square$ at $p_{\text {dep }}=4 \times 10^{-5} \mathrm{mbar}$ to $870 \Omega / \square$ at $1 \times 10^{-2}$ mbar. For each sample, a slight upturn in resistance is observed at low temperatures, while the resistance minimum shifts systematically with $p_{\text {dep }}$ from about $4 \mathrm{~K}$ for $p_{\text {dep }}=4 \times 10^{-5}$ mbar to about $10 \mathrm{~K}$ for $p_{\text {dep }}=1 \times 10^{-2}$ mbar [Fig. 1(b)]. In the literature, this behavior has been termed Kondo-like, while the details of the involved scattering mechanism are still under debate $[5,64]$.

Figure 2(a) shows typical Hall data, in this case obtained for the sample grown at $p_{\text {dep }}=1 \times 10^{-4}$ mbar at selected temperatures for external fields up to $\pm 13 \mathrm{~T}$. The Hall effect is linear at 300 and $100 \mathrm{~K}$, while it shows a clear nonlinear dependence on magnetic field at 30,10, and $2 \mathrm{~K}$. This nonlinear Hall effect is attributed to multiple carrier conduction that has been reported both experimentally $[16,65]$ as well as discussed theoretically [17] for (a)

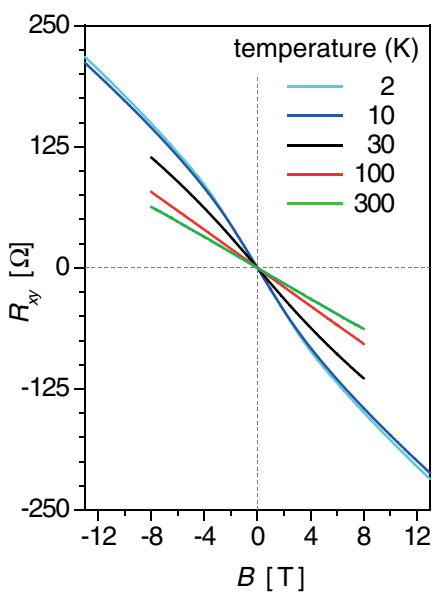

(b)

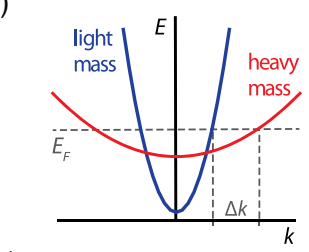

(c)

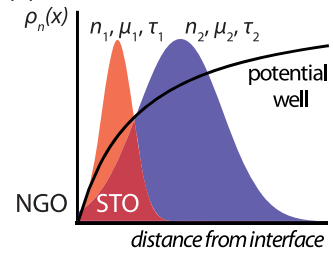

FIG. 2. (a) Antisymmetrized Hall resistance $R_{x y}(B)$ obtained for the NGO/STO heterostructure grown at $p_{\text {dep }}=1 \times 10^{-4} \mathrm{mbar}$. (b) In the band structure schematic (after Refs. [16,17]), electrons have distinct effective masses $m_{i}^{*}$ for light and heavy mass bands. (c) In the defect inhomogeneity scenario, electrons experience distinct scattering times $\tau_{i}$, as distributions are located at different distances from the interface. 
LAO/STO. One origin of this multiple carrier conduction discussed in the literature is the complex band structure of STO comprising light and heavy mass bands crossing the Fermi level [14-17] [see Fig. 2(b)]. At low temperatureswhere interband scattering is generally suppressed due to the required momentum transfer-electrons in these bands act as separated populations contributing to transport with different effective masses $m_{i}^{*}$.

Alternatively, one may consider that the different carrier populations within the potential well at the NGO/STO interface have different distributions $\rho_{i}$ in their distance from the interface [Fig. 2(c)]. The defect structure is inhomogeneous when traversing from regions close to the interface to regions farther away from the interface [13]. This will induce different mean scattering times $\tau_{i}$, leading to different mobilities, $\mu_{i}=e \tau_{i} / m_{i}^{*}$, of the various charge carrier populations at low temperatures.

As a result of multiple carrier conduction, one derives a nonlinear field dependence of the Hall resistance in a semiclassical approach considering two-electron populations. The Hall resistance is then given by

$$
R_{x y}^{2 e}=-\frac{1}{e} \frac{\left(\frac{n_{1} \mu_{1}^{2}}{1+\mu_{1}^{2} B^{2}}+\frac{n_{2} \mu_{2}^{2}}{1+\mu_{2}^{2} B^{2}}\right) B}{\left(\frac{n_{1} \mu_{1}}{1+\mu_{1}^{2} B^{2}}+\frac{n_{2} \mu_{2}}{1+\mu_{2}^{2} B^{2}}\right)^{2}+\left(\frac{n_{1} \mu_{1}^{2}}{1+\mu_{1}^{2} B^{2}}+\frac{n_{2} \mu_{2}^{2}}{1+\mu_{2}^{2} B^{2}}\right)^{2} B^{2}} .
$$

This model is used to fit the nonlinear Hall data under the constraint

$$
R_{x x}^{-1}(B=0)=e\left(n_{1} \mu_{1}+n_{2} \mu_{2}\right)\left[+R_{K}^{-1}(B=0)\right] .
$$

Here, $R_{K}$ denotes a small Kondo correction term that may enter into the zero-field resistance, related to the slight resistance increase observed at low temperature. For further analysis, the Kondo term is neglected. Note that any nonzero Kondo contribution in this boundary condition (taken at $B=0$ ) enters only into the error estimation of the transport parameters (as a function of temperature). It does not affect the anomalous $B$-field behavior of $R_{x y}$ discussed in the remainder of this study.

Using the high-field data only, the two-carrier model delivers reasonable fits (dashed lines) to the experimental data obtained for $R_{x y}$ as depicted in Fig. 3(a). Both carrier types have electron character.

However, the Hall coefficient, $R_{H}=R_{x y} / B$, reveals a systematic deviation between the fit and the experimental data observed at low temperatures $(\lesssim 10 \mathrm{~K})$ around zero field [see Fig. 3(b)]. As we show in Fig. 3(b) as well as in the enlarged view in Fig. 4(a), $R_{H}$ shows an upturn at low fields. This unusual feature is evident for fields up to about $\pm 2.5 \mathrm{~T}$ and is, thus, clearly distinct from the typical scatter observed in the Hall coefficient near zero field. A similar feature has also been observed for LAO/STO $[15,16]$.

Within the two-carrier (2e) model, $R_{H}^{2 e}$ follows a Lorentzian-like shape with a single minimum at zero field
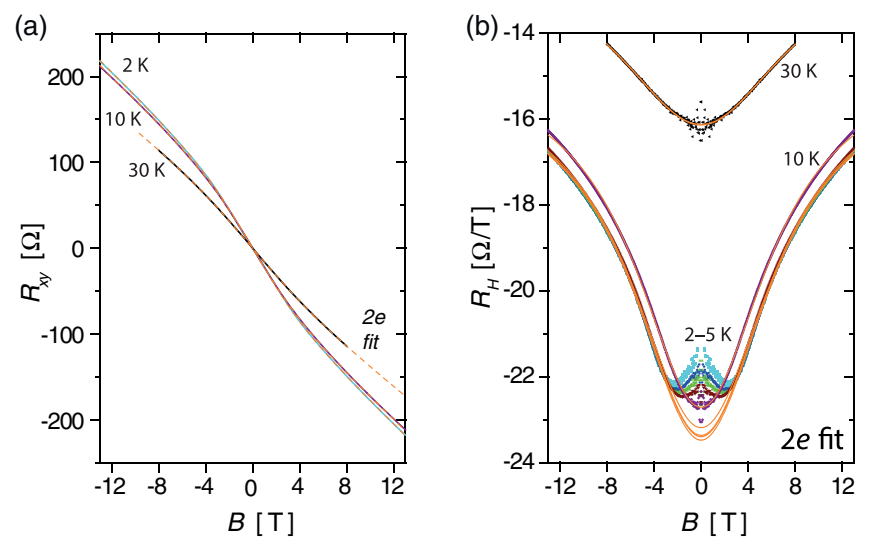

FIG. 3. Hall characteristics obtained for the sample grown at $p_{\text {dep }}=1 \times 10^{-4}$ mbar in the low-temperature regime for external fields up to $\pm 13 \mathrm{~T}$. (a) $R_{x y}$ obtained from experiment including data fits according to the two-electron model ( $2 e$, dashed lines) for $T=2,10$, and $30 \mathrm{~K}$. (b) Hall coefficient $R_{H}=R_{x y} / B$ obtained from the data in (a) for $T=2,3,4,5,10$, and $30 \mathrm{~K}$.

[orange lines in Fig. 4(a)], so that it does not capture the experimentally observed field dependence of $R_{H}$ at all. Thus, a mere two-electron model fails to accurately explain the Hall data observed in our heterostructures at temperatures below about $10 \mathrm{~K}$.

In order to account for the lower-field behavior of the Hall coefficient, we incorporate an additional correction term describing an anomalous Hall component indicating the presence of magnetic ordering at the NGO/STO interface. The experimental data are then decomposed as the sum of two contributions, from two-carrier conduction and the AHE:

$$
R_{x y}=R_{x y}^{2 e}+R_{x y}^{\mathrm{AHE}}=R_{x y}^{2 e}+R_{0}^{\mathrm{AHE}} M_{z}(B) .
$$
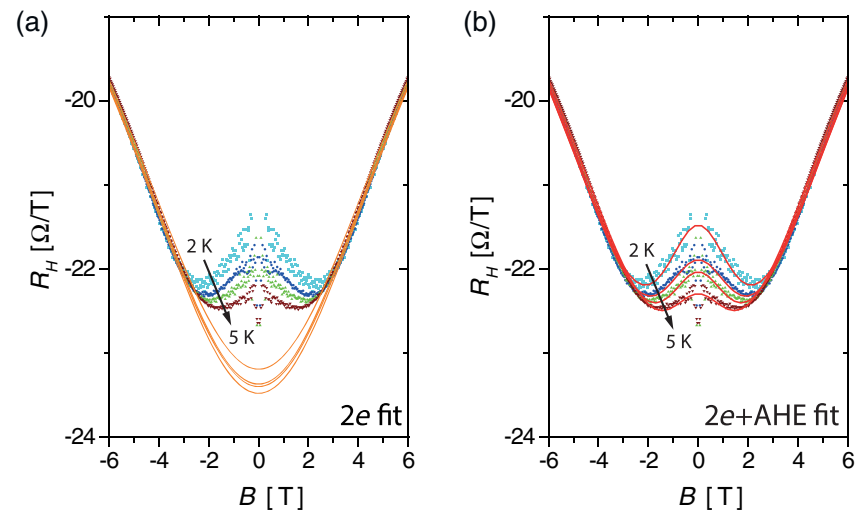

FIG. 4. (a) $R_{H}$ at low fields $( \pm 6 \mathrm{~T})$ for the sample grown at $p_{\text {dep }}=1 \times 10^{-4}$ mbar. Different colors of data points correspond to temperatures of $2,3,4$, and $5 \mathrm{~K}$. The fits according to the $2 e$ model (solid lines) show a clear deviation from the experiment around zero field. (b) Same data as displayed in (a) now including fits according to the $2 e$ model with an additional AHE term (red solid lines). 
Here, $M_{z}$ represents a possible magnetization in the $z$ direction present at the NGO/STO interface. Any spontaneous magnetization is expected to be oriented within the interface plane due to the strong shape anisotropy, as observed in LAO/STO $[9,25,66]$. Thus, ordered moments at the NGO/STO interface should rotate around a hard axis when applying an external magnetic field perpendicular to the interface in the standard Hall geometry. For this reason, $M_{z}$ is expected to vary smoothly when sweeping $B$ from negative to positive values, finally saturating above a critical field $B_{c}$. Notably nonhysteretic behavior would be expected in this case, in agreement with our experiment. Such a field behavior can be described phenomenologically by a Langevin-type function comprising two free parameters, $B_{c}$ and $R_{0}^{\mathrm{AHE}}$. With these considerations, we get

$$
R_{x y}^{\mathrm{AHE}}=R_{0}^{\mathrm{AHE}} \tanh \left(\frac{B}{B_{c}}\right)
$$

with $R_{0}^{\mathrm{AHE}}$ being proportional to the saturation magnetization, $R_{0}^{\mathrm{AHE}} \propto M_{0}$. This nonhysteretic functional form is consistent with the AHE observed in magnetic oxides such as $(\mathrm{La}, \mathrm{Sr}) \mathrm{MnO}_{3}$ [67,68] or in metallic Co thin films $[40,69]$.

As we show in Fig. 4(b), the assumption of an additional AHE component in the Hall effect in NGO/STO leads to a significantly improved fit (red lines) reproducing the behavior of both $R_{x y}$ and $R_{H}$ over the entire field range. In particular, the anomalous behavior observed around zero fields is fully captured by the model. The remaining deviations near zero field can be attributed to experimental noise being amplified in the ratio $R_{x y} / B$ for small $B$.

As we show in Fig. 5(a), a similar anomalous behavior of $R_{H}$ is found for all samples. The width of the anomalous upturn around zero field increases with increasing $p_{\text {dep }}$, as indicated by the gray boxes. For the highest growth pressure, the AHE generates a very broad feature that at the lowest temperatures $(\lesssim 5 \mathrm{~K})$ dominates the $R_{H}$ characteristics over a wide field range. In this case, our fitting has to be stabilized by keeping one transport parameter, here $\mu_{2}$, fixed to its value at $10 \mathrm{~K}$. For all samples, the suggested model comprising two-electron transport and an anomalous Hall component reproduces the behavior of the Hall coefficient over the entire field range [Fig. 5(b)], whereas the two-electron model alone fails. The good agreement between the experimental data and the analysis therefore suggests that there are two contributions in the Hall effect at low temperatures: (1) a contribution due to multiple carrier conduction $(T \lesssim 30 \mathrm{~K})$ and $(2)$ a contribution due to the AHE indicating magnetic ordering $(T \lesssim 10 \mathrm{~K})$.

Generally, one may also consider alternative routes other than magnetism to accommodate the observed anomalous feature in the Hall coefficient characteristics. One obvious way would be the consideration of additional electron populations (i.e., $n_{3}, \mu_{3}, n_{4}, \mu_{4}, \ldots$ ) as predicted by Khalsa

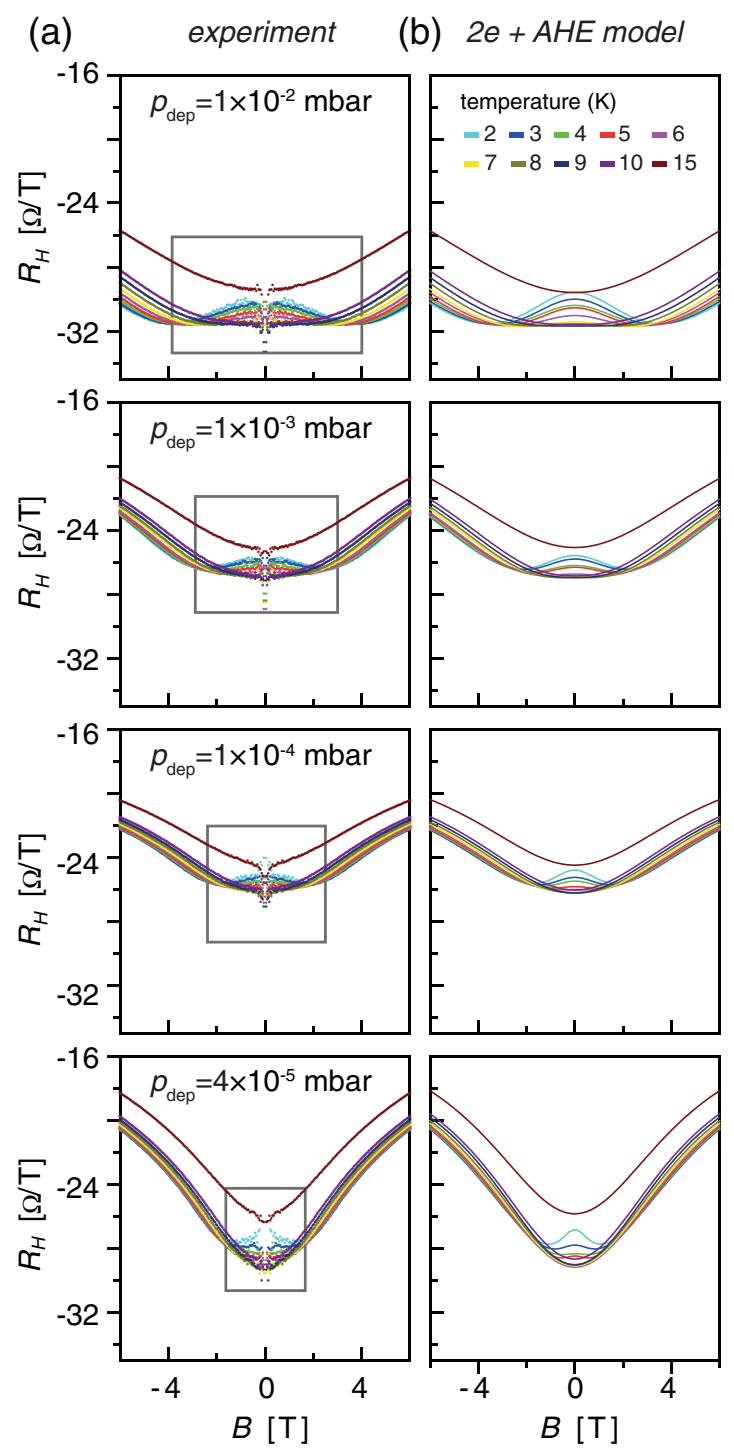

FIG. 5. (a) Antisymmetrized Hall coefficient obtained experimentally for various temperatures between 2 and $15 \mathrm{~K}$. Gray boxes are guides to the eye approximately indicating the field range of the AHE. (b) Corresponding data fits using the extended model considering two electron populations and an AHE contribution.

and MacDonald [17]. However, as discussed in Ref. [43], as many as ten electron populations do not significantly improve the fit to the data-in particular, in the lowfield range. Likewise, field-dependent electron mobilities did not reproduce this feature [43]. The inclusion of mobile holes in the fitting is another way to induce an upturn in the Hall coefficient characteristics. However, significant low-temperature hole conduction is rather unlikely in STO: even nominally undoped STO naturally comprises a reservoir of ionized acceptor-type impurities. These impurities neutralize at low temperatures and suppress any $p$-type conduction [70]. Moreover, the hole scenario yields unrealistically high hole mobility values, ruling out any $p$-type 

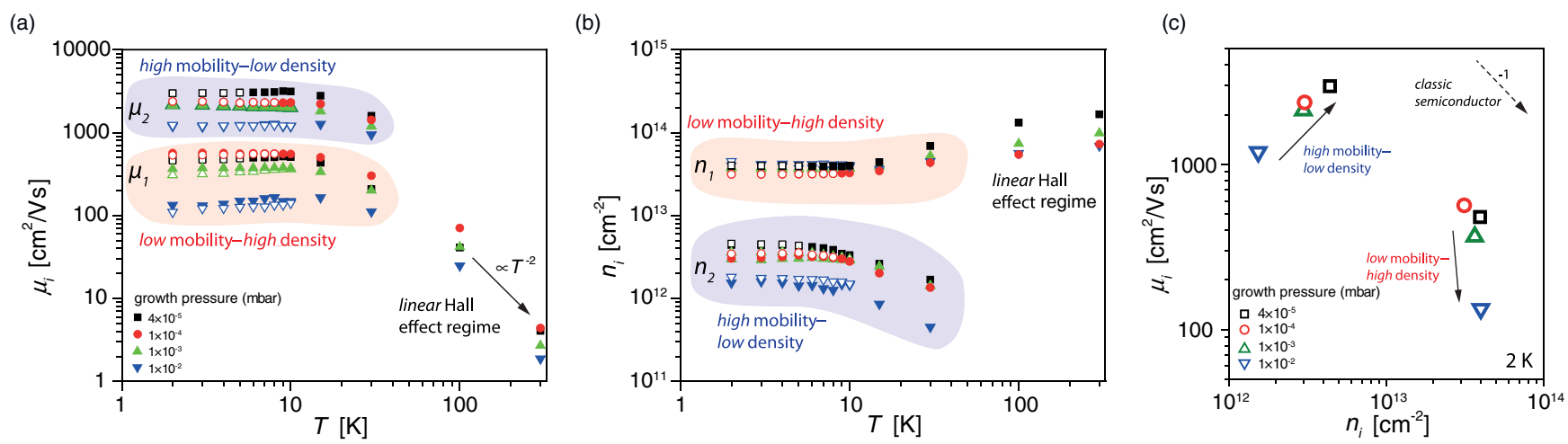

FIG. 6. Transport parameters for NGO/STO heterostructures grown at various deposition pressures. (a), (b) Electron mobility $\mu_{i}$ and sheet electron density $n_{i}$ of the two electron populations. One can identify a low-mobility-high-density population and a high-mobilitylow-density population. Different symbols of the same color indicate the parameters obtained from the two-electron model with (open) and without (filled) the AHE term. (c) Electron mobility obtained at $2 \mathrm{~K}$ (defect scattering regime) as a function of $n_{i}$. The dashed line indicates the expected behavior for a classical $n$-type semiconductor.

conduction in the polar capping layer [29,71]. The assumption of magnetism, hence, seems the most likely scenario.

As illustrated in Figs. 6(a) and 6(b), the obtained best fits for the parameters $n_{i}$ and $\mu_{i}$ are essentially unchanged for the fit including the AHE contribution (open symbols) and without it (filled symbols). This implies that the assumption of an additional term due to the AHE does not contradict or significantly affect the physics of electron transport with multiple electron contributions already discussed in the literature [14-17,65].

Using this two-carrier model with AHE, we can now extract the parameters for both the electronic transport and the AHE as a function of the growth conditions. Based on these values, we discuss the possible defect structure, electronic properties, and magnetic properties of the $\mathrm{NGO/STO}$ interface in the next section.

\section{B. Behavior of electronic transport for different growth parameters}

Figure 6 shows the transport parameters, $\mu_{1}, \mu_{2}$ [Fig. 6(a)] and $n_{1}, n_{2}$ [Fig. 6(b)], extracted from the fits of the Hall data as a function of temperature for samples grown at various $p_{\text {dep }}$. In accordance with previous reports, we can identify a high-density-low-mobility electron population and a low-density-high-mobility electron population at low temperatures. The mobility values saturate at low temperature, indicating a mean scattering time $\tau_{i}$ dominated by defect scattering. At moderate temperatures ( $\gtrsim 30 \mathrm{~K}$ ), the two-electron channels are no longer distinguishable (linear Hall effect), presumably due to interband scattering and a common $\tau_{i}$ dominated by electron-electron scattering. In agreement with this, the mobility values show the typical temperature dependence $\mu \propto T^{-2}$ reported for LAO/STO heterostructures [72] in this temperature range.
With respect to defect structure, the low-temperature regime is especially interesting. As shown in Fig. 6(a), the mobility values of both electron populations generally decrease with increasing $p_{\text {dep }}$, in agreement with the observed behavior of the sheet resistance [Fig. 1(a)]. The high-mobility electron population exhibits mobility values between $3000 \mathrm{~cm}^{2} / \mathrm{V}$ s for $p_{\text {dep }}=4 \times 10^{-5} \mathrm{mbar}$ and $1200 \mathrm{~cm}^{2} / \mathrm{V} \mathrm{s}$ at $p_{\text {dep }}=1 \times 10^{-2}$ mbar which is in the typical range reported for LAO/STO heterostructures $[41,73]$. Likewise, the low-mobility electron population exhibits mobility values between $500 \mathrm{~cm}^{2} / \mathrm{V} \mathrm{s}$ at a lower $p_{\text {dep }}$ and $130 \mathrm{~cm}^{2} / \mathrm{V} \mathrm{s}$ at the highest $p_{\text {dep }}$.

Interestingly, the carrier concentrations obtained for the high-mobility population $\left[n_{2} \approx(1-5) \times 10^{12} \mathrm{~cm}^{-2}\right]$ are of the same order as the carrier densities typically obtained from quantum oscillations in LAO/STO [74,75], strongly suggesting that only this particular high-mobility electron population contributes to quantum transport. However, given the limitations of the two-carrier fitting with the AHE term, as we discuss in Sec. IV. A, we cannot determine a full subband picture in the absence of quantum oscillation measurements (which have vanishing amplitude at these high total carrier densities).

The $\mu_{2} / \mu_{1}$ ratio $(\approx 4-6)$ for a particular NGO/STO interface may be understood in terms of the reported effective masses of the light and heavy mass bands in STO [cf. Fig. 2(b)]. However, the variation of the electron mobility of a given population as well as the variation of $\mu_{2} / \mu_{1}$ from sample to sample can be understood only in terms of a varying defect structure in the interface region. Therefore, the observed decrease of the mobility values with increasing $p_{\text {dep }}$ has to be attributed to a varied defect structure in the potential well established during growth. As oxide interfaces generally have to be considered as vertically inhomogeneous systems, effects due to a change in the shape of the potential well (as reported for gated 

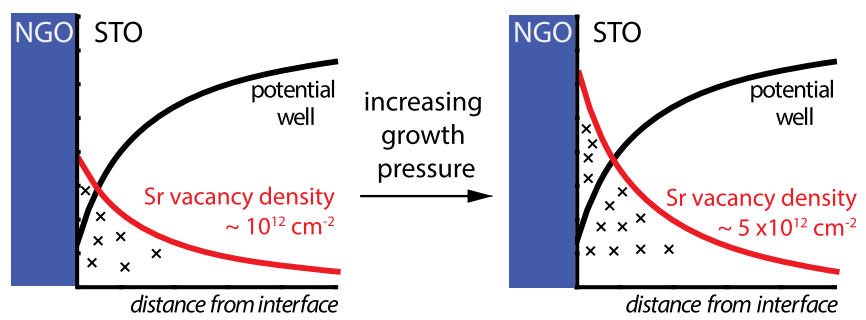

FIG. 7. Schematic of the defect structure at the NGO/STO interface for different $p_{\text {dep }}$ based on low-temperature electron mobility and carrier density. The shape of the potential well is assumed to be similar in both cases, while the density of defects increases with increasing $p_{\mathrm{dep}}$. In particular, the concentration of cation vacancies increases for higher $p_{\text {dep }}$, mediated by the Schottky equilibrium.

interfaces [65]) and effects due to distinct defect structures have to be separated. As shown in Fig. 6(b), however, the high carrier concentration, and thus the total carrier concentration, $n=n_{1}+n_{2}$, is rather similar for all samples $\left[\sim(4 \pm 1) \times 10^{13} \mathrm{~cm}^{-2}\right.$ at $\left.2 \mathrm{~K}\right]$. Therefore, the screening length of the potential well (scaling with $n^{-1 / 2}$ ) and thus the relative location of the electron gas should be comparable for all samples, assuming a purely electronic picture in which electrons are the majority charge carriers within the well $[76,77]$. Hence, the actual defect structure inside the potential well at the NGO/STO interface must differ in the samples grown at different deposition pressure. In particular, the behavior of the low-temperature mobility indicates an increasing defect density with increasing $p_{\text {dep }}$ (Fig. 7).

Keeping in mind that growth dynamics in PLD processes change dramatically with $p_{\text {dep }}$ [46], it may not be surprising that the defect structure of the NGO films and hence the NGO/STO interface varies from sample to sample. The observed trend of the electron mobility, however, allows a more detailed discussion of the interfacial defect structure - in particular, the character of the STO adjacent to the interface which is most important for electron transport.

Figure 6(c) displays the electron mobility obtained in the defect-scattering-dominated temperature regime $(2 \mathrm{~K})$ as a function of sheet carrier density. The high-mobility-lowdensity electron population shows an increase in mobility with increasing carrier density, while the low-mobilityhigh-density electron population shows a steep decrease in mobility at almost constant carrier density.

Both trends do not agree with a classical donor-doping scenario in semiconductors, for which one expects $\mu \propto\left[D^{*}\right]^{-1} \propto n^{-1}$, where $\left[D^{*}\right]$ denotes the dopant concentration. For a constant extension of the doped region (here, for similar screening lengths), this maps into $\mu \propto n_{S}^{-1}$ (gray arrow). It is thus unlikely that the concentrations of donortype defects, i.e., oxygen vacancies $\mathrm{V}_{\mathrm{O}}^{*}$, or extrinsic dopants such as $\mathrm{Nd}^{3+}$ cations substituting $\mathrm{Sr}^{2+}$ are responsible for the observed trend. We find the highest mobility values for the samples grown at low pressures, where both the generation of oxygen vacancies as well as kinetic intermixing of cations (also $B$-site intermixing [78] possibly resulting in acceptor-type $\mathrm{Ga}$ /Ti-antisite defects) should be most pronounced [19]. Hence, the dependence on $p_{\text {dep }}$ is not consistent with a scenario based on donor-type defects, such as similarly reported in Ref. [79] for LAO/STO.

In summary, we can, therefore, rule out that oxygen vacancies are the mobility-limiting defects in our samples (via thermodynamic considerations). Moreover, we can rule out cation intermixing and antisite defects (via kinetics considerations) as well as the shape of the potential well (given the fixed total carrier density) as responsible for the observed behavior of the electron mobility.

Instead, one has to consider defects arising predominantly at higher $p_{\text {dep }}$ and having acceptor-type character. The clear defect candidate for this is a strontium vacancy $\mathrm{V}_{\mathrm{Sr}}^{\prime \prime}$. As reported in the literature [18,20,21,32,80], these are readily induced at oxide interfaces during growth and annealing procedures [24], such as indicated, e.g., by observation of $\mathrm{SrO}$ segregation during growth of LAO (and NGO) [34]. The Schottky equilibrium is active during the PLD growth process $[21,54,80]$, so that the variation of the $p_{\text {dep }}$ affects the strontium vacancy concentration present at the NGO/STO interface. In fact, a defect scenario based on the formation of $\mathrm{V}_{\mathrm{Sr}}^{\prime \prime}$ is fully consistent with the behavior of $\mu_{i}$ as a function of both $n_{i}$ and $p_{\mathrm{dep}}$. (1) $\mathrm{V}_{\mathrm{Sr}}^{\prime \prime}$ are increasingly formed upon oxidation at relatively high temperatures, which agrees with the decrease in electron mobility for increasing $p_{\text {dep }}$. (2) $\mathrm{V}_{\mathrm{Sr}}^{\prime \prime}$ are acceptor-type defects consistent with a decrease in electron mobility for decreasing carrier density as observed for $n_{2}$. Strontium vacancies may therefore be considered the dominant defects at the interface of samples grown at high oxygen pressure, while oxygen vacancies and cation intermixing are less important here.

Other defects in the NGO thin film itself are expected to have a major influence on the carrier density rather than on the electron mobility, which should be affected solely by defects on the STO side of the interface. In particular, cation nonstoichiometry in the polar oxide layer should diminish the interfacial dipole, resulting in a reduced carrier density $[32,81,82]$. Here, however, the total carrier density is primarily unchanged, so that we can exclude a severe effect of nonstoichiometry in our experiments. Nevertheless, we cannot exclude a minor effect caused by a variation of the $\mathrm{Ga}$ content in films grown at high $p_{\text {dep }}$ [52].

Besides acting as scattering centers, strontium vacancies are acceptor-type charged defects reducing the electron density at the interface $[18,20]$. As shown in Fig. 6(b), however, only the density of the high-mobility electron population $\left(n_{2}\right)$ is significantly reduced in the samples grown at increased growth pressure, while the density of the low-mobility population $\left(n_{1}\right)$ is unchanged. Hence, the 
sheet concentration of strontium vacancies is likely to vary on the same order of magnitude as $n_{2}$ in these samples. This corresponds to a strontium vacancy sheet density in the range of (or below) $1 \times 10^{12} \mathrm{~cm}^{-2}$ for the sample grown at $4 \times 10^{-5} \mathrm{mbar}$, and about $5 \times 10^{12} \mathrm{~cm}^{-2}$ for the sample grown at $1 \times 10^{-2}$ mbar. This result is consistent with previous studies probing the defect chemistry of the $2 \mathrm{DEG}$ system in thermodynamic equilibrium [18,20,32]. Quantitatively, equilibrium defect chemistry predicts an increase of the strontium vacancy concentration by about 1 order of magnitude for an increase of the growth oxygen partial pressure by 2 orders of magnitude $\left[\log \left[\mathrm{V}_{\mathrm{Sr}}^{\prime \prime}\right] \propto\right.$ $\left.1 / 2 \log \left(p \mathrm{O}_{2}\right)\right][13,53,55]$, which is consistent with the observed trend. Given the estimated sheet concentration of $\mathrm{Sr}$ vacancies, most of the charge accumulated in the potential well at the NGO/STO interface is accommodated by the electron gas. Only a fraction $(\lesssim 10 \%-20 \%)$ of the total charge is compensated by ionic $\mathrm{Sr}$ vacancies. Hence, the shape of the potential well at the interface is mainly determined by the mobile electrons [13]. As a result, the interface potential well in our samples remains in comparable shape although the ionic background defect structure varies among the samples, resulting in the observed decrease in electron mobility at increased growth pressure.

\section{Scaling of the AHE component with growth parameters}

Turning to the AHE contribution, we separate in Fig. 8(a) the two Hall components, $R_{x y}^{2 e}$ and $R_{x y}^{\mathrm{AHE}}$, extracted from the data fit at $2 \mathrm{~K}$ for the sample grown at $1 \times 10^{-4}$ mbar. $R_{x y}^{\mathrm{AHE}}$ (solid line) is only a small contribution to the total Hall resistance (solid red line), while the major part of the Hall voltage drop is due to the conventional two-carrier conduction $R_{x y}^{2 e}$ (dashed line). The anomalous Hall contribution exhibits opposite sign as the conventional contribution, taking positive values at positive fields and negative values at negative fields, which is similar to the AHE observed in metallic Co $[40,69]$.

In Fig. 8(b), we plot the field dependence of $R_{x y}^{\mathrm{AHE}}$ extracted from the fitting at $2 \mathrm{~K}$ for the samples prepared under varied growth conditions. $R_{x y}^{\mathrm{AHE}}$ scales systematically with $p_{\text {dep }}$. The largest AHE contribution is found for the sample grown at $1 \times 10^{-2}$ mbar with $R_{0}^{\mathrm{AHE}} \approx 20 \Omega$ (corresponding to about $10 \%$ of the total Hall resistance at $10 \mathrm{~T}$ ), the smallest contribution for the sample grown at $4 \times$ $10^{-5}$ mbar with $R_{0}^{\mathrm{AHE}} \approx 4 \Omega$ (about $2 \%$ of the total Hall resistance at $10 \mathrm{~T})$ at $2 \mathrm{~K}$. Thus, $R_{0}^{\mathrm{AHE}}\left(\propto M_{0}\right)$ roughly scales by a factor of 5, which is of the same order as the scaling of $R_{x x}$ [Fig. 1(a)] and $\mu_{i}$ [Fig. 3(b)]. This is illustrated in Fig. 9, which plots $R_{0}^{\mathrm{AHE}}$ obtained at $2 \mathrm{~K}$ as a function of the residual resistance $R_{x x}$, revealing a linear relationship. This scaling behavior is consistent with the general theory of AHE [36]. In particular, a linear scaling indicates an extrinsic anomalous Hall effect, pointing towards a defect-controlled mechanism.
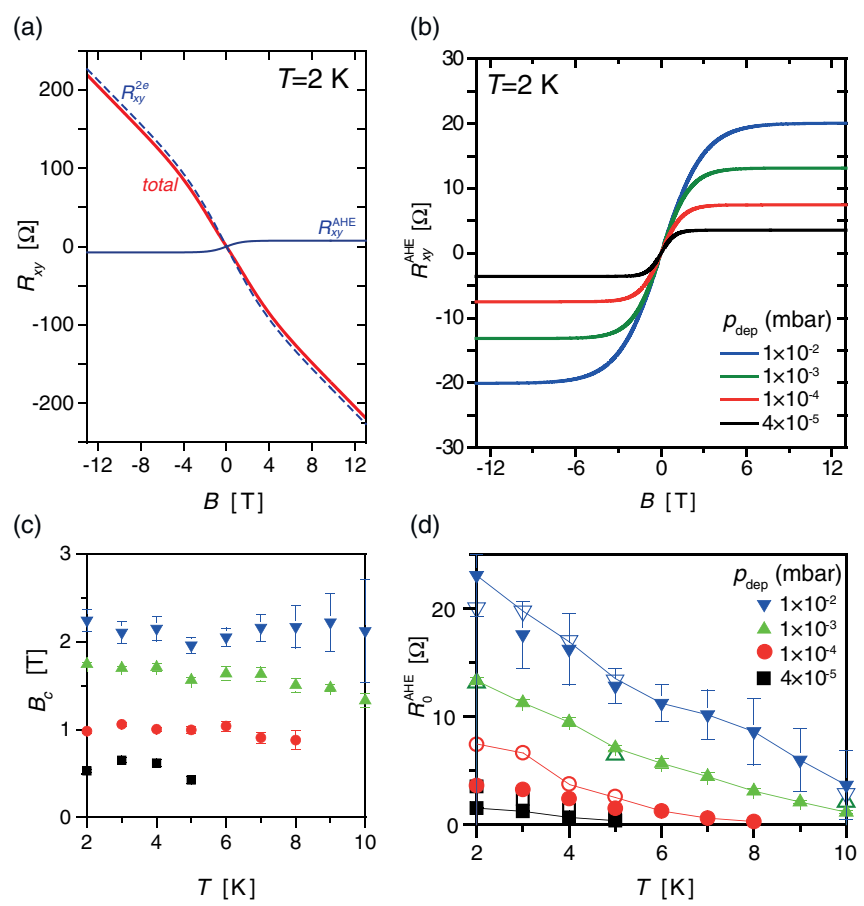

FIG. 8. (a) Separation of conventional $2 e$ contribution, $R_{x y}^{2 e}$ (dashed line), and the $\mathrm{AHE}$ contribution $R_{x y}^{\mathrm{AHE}}$ (solid line), obtained from fitting (solid red line) the total Hall resistance $R_{x y}$ (respectively, the Hall coefficient $R_{H}$ ) at $2 \mathrm{~K}$ for the sample grown at $p_{\text {dep }}=1 \times 10^{-4}$ mbar. (b) Comparison of the AHE contribution obtained for different deposition pressure. (c) Critical field $B_{c}$ for saturation of the AHE contribution as a function of temperature. (d) Saturation resistance, $R_{0}^{\mathrm{AHE}} \propto M_{0}$, of the AHE contribution as a function of temperature and deposition pressure. The critical temperature $T_{c}$ is defined by $R_{0}^{\mathrm{AHE}} \rightarrow 0$ to within experimental noise.

The critical field $B_{c}$ at which the AHE component starts to saturate scales with $p_{\text {dep }}$, shifting from about $0.5 \mathrm{~T}$ at $4 \times 10^{-5}$ mbar to $2.2 \mathrm{~T}$ at $1 \times 10^{-2}$ mbar [Fig. 8(b)]. This increase corresponds to the broadening of the anomalous hump observed in the Hall coefficient characteristics [cf. gray boxes in Fig. 5(a)]. Hence, the external field required to align all apparent interfacial magnetic moments perpendicular to the interface $\left(B \approx B_{c}\right)$ scales with $p_{\text {dep }}$.

Figures 8(c) and 8(d) show the temperature dependence of $B_{c}$ and $R_{0}^{\mathrm{AHE}}$, respectively. While the width of the AHE contribution, $B_{c}$, is temperature independent in all samples, the amplitude of the AHE, $R_{0}^{\mathrm{AHE}}$, decreases with increasing temperature, vanishing at temperatures between $5 \mathrm{~K}$ for the sample grown at $p_{\text {dep }}=4 \times 10^{-5} \mathrm{mbar}$ and $\gtrsim 10 \mathrm{~K}$ for the samples grown at higher $p_{\text {dep }}$. The critical temperature $T_{c}$ for the AHE to arise hence also scales with growth parameters, showing a similar trend as the temperature at which a minimum in the $R_{x x}(T)$ is observed [cf. Fig. 1(a)].

The measurements are repeated using different experimental setups [open and closed symbols in Figs. 8(d) and 9]. As shown in Fig. 8(d), both the amplitude and the 


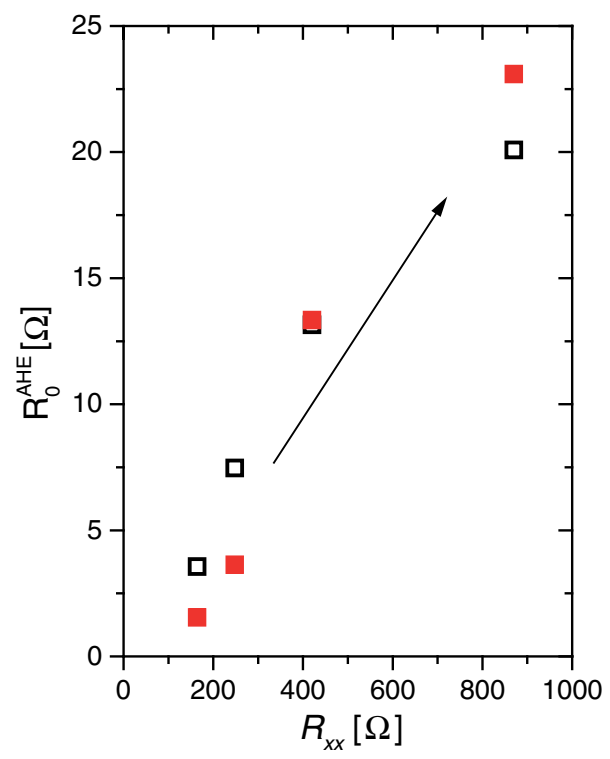

FIG. 9. $R_{0}^{\mathrm{AHE}}$ obtained at $2 \mathrm{~K}$ as a function of the residual resistance $R_{x x}$ revealing a linear relationship. Open and closed symbols indicate measurements repeated using different experimental setups (cf. Fig. 8).

temperature dependence of the AHE are generally similar in both measurements.

The Langevin function used to describe the anomalous Hall component in the suggested model commonly describes a paramagnetic spin- $1 / 2$ system, with $B_{c}$ being proportional to $k_{B} T$ and $R_{0}^{\mathrm{AHE}} \propto M_{0}$ being temperature independent. Contrary to this, we find that $B_{c}$ is rather temperature independent, while $R_{0}^{\mathrm{AHE}}$ decreases with increasing temperature until the AHE component disappears at $T_{c}$. We can, therefore, rule out a paramagnetic behavior of the apparent magnetism at the NGO/STO interface as observed here. In contrast, for coupled magnetic moments the saturation magnetization $M_{0}$ and, thus, $R_{0}^{\mathrm{AHE}}$ should decrease with increasing temperature close to the Curie temperature. Thus, the observed behavior is consistent with the existence of weakly coupled or ferromagnetic moments at the NGO/STO interface, in agreement with previous reports on magnetism in LAO/STO $[6,7,9,25]$. The critical temperature for the observation of an anomalous Hall component may then be interpreted as the ordering temperature. The related energy scale $k_{B} T_{c}$ is of the order of $0.4-1 \mathrm{meV}$, indicating a weak coupling of the magnetic moments. In this scenario, the functional form of the Langevin function has only limited physical meaning and serves only as a useful mathematical description consistent with experimental observations. As shown in Fig. 8(d), the fitting parameter $B_{c}$ increases with increasing $p_{\text {dep }} . B_{c}$ may be connected to the strength of the demagnetizing fields in a particular sample. However, care has to be taken as the absolute values of $B_{c}$ are rather high, perhaps too high to be assigned to demagnetizing fields.

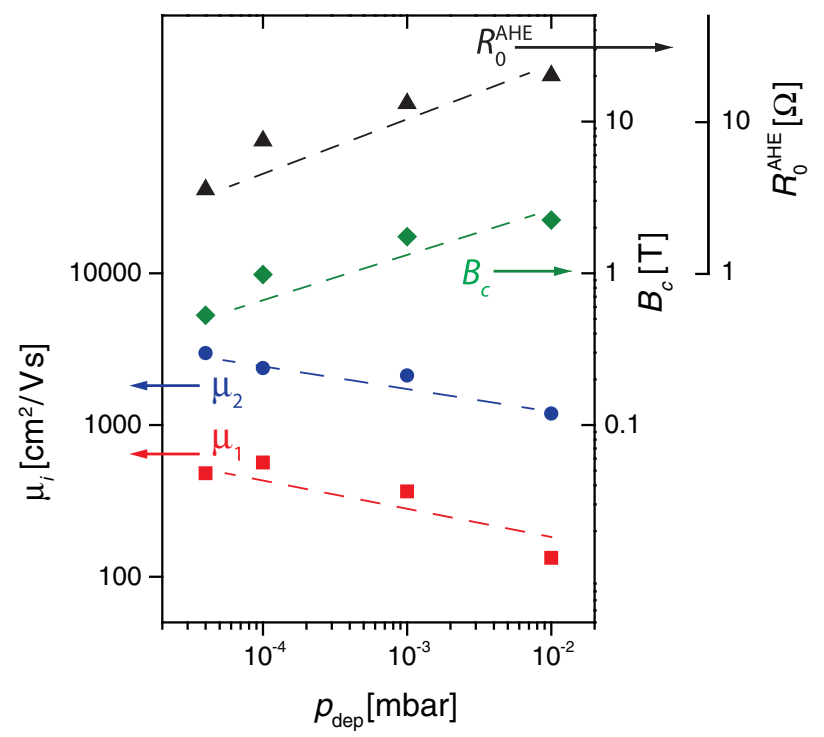

FIG. 10. Summary of anomalous and conventional transport parameters obtained at $2 \mathrm{~K}$ as a function of $p_{\text {dep }}$. While the quantities characterizing the AHE increase with increasing $p_{\text {dep }}$, the electron mobility characterizing the defect structure shows the inverse dependence on $p_{\mathrm{dep}}$. The dashed lines are guides to the eye.

Note, however, that torque magnetometry [6] also revealed a torque response at fields above $1 \mathrm{~T}$ in LAO/STO, which may be consistent with the high values found for $B_{c}$ in this study.

The systematic trend with $p_{\text {dep }}$ found for all AHE parameters, $R_{0}^{\mathrm{AHE}}, B_{c}$, and $T_{c}$, again suggests a correlation of electronic-ionic structure and magnetic properties of the NGO/STO interface. As the AHE scales with resistance and electron mobility rather than with electron concentration, it seems that the ionic structure governing the electron mobilities is the most significant factor here. In particular, the most defective samples with the lowest mobility values and highest sheet resistance values exhibit the most pronounced anomalous Hall effect contribution, as illustrated in Fig. 10. Here, we plot $\mu_{i}, B_{c}$, and $R_{0}^{\mathrm{AHE}}$ as a function of growth pressure on logarithmic scale. Clearly, the quantities characterizing the anomalous Hall effect show the inverse dependence on $p_{\text {dep }}$ as the electron mobility characterizing the interfacial defect concentration. While the scaling behavior of $R_{0}^{\mathrm{AHE}}$ may be understood as a direct result of the decreased electron mobility in the samples grown at high deposition pressure, the scaling of $B_{c}$ and $T_{c}$ indicates that the inherent magnetic properties of the interface change with increasing concentration of $\mathrm{Sr}$ vacancies.

Consequently, the treatment of the AHE as discussed within this study suggests (1) the presence of coupled magnetic moments at the NGO/STO interface arising at temperatures $\lesssim 10 \mathrm{~K}$ and (2) tunability of the magnetic properties through the control of the interfacial defect structure. 

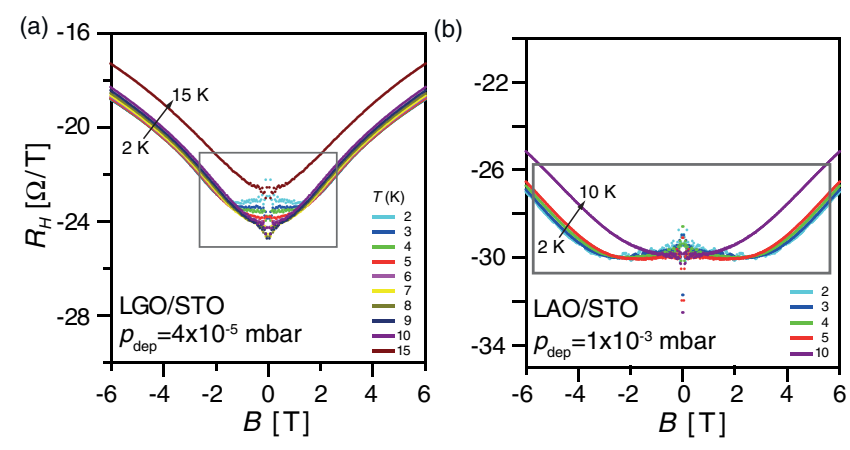

FIG. 11. Anomalous Hall effect observed in (a) LGO/STO grown at $p_{\text {dep }}=4 \times 10^{-5}$ mbar and (b) LAO/STO grown at $p_{\text {dep }}=1 \times 10^{-3}$ mbar. Gray boxes are guides to the eye roughly indicating the field range of the AHE.

\section{Comparison to other heterostructure systems and the role of intrinsic defects}

A special concern for magnetism in oxides is the effect of magnetic impurities mimicking magnetic moments in nominally nonmagnetic materials. However, the sole effect of extrinsic impurities cannot explain the systematic trend with growth parameters observed in this study. Moreover, we observe the largest AHE for the sample with the lowest oxygen vacancy concentration ( $p_{\text {dep }}=1 \times 10^{-2} \mathrm{mbar}$ ). Thus, a relation of the observed AHE with oxygen vacancies as proposed as the origin of magnetism in LAO/STO [26,30] is unlikely. Similarly, the formation of antisite defects [78] should be least pronounced in this sample.

Another aspect is the role of the $\mathrm{Nd} 4 f$ electrons that carry a magnetic moment $[35,83]$ and through which magnetism may be induced into the NGO/STO interface either via intermixing or via proximity effects. However, as shown in Fig. 11, we observe a similar AHE also for LAO/ STO and LGO/STO heterostructures, both of which do not contain Nd. Moreover, the anomalous Hall effect as discussed within this study has been observed before in similar magnitude in the absence of Nd ions in LAO/STO and other STO-based 2D electron systems $[15,16,43]$. In fact, the AHE does not seem to be significantly enhanced in NGO/STO as compared to other systems not containing $\mathrm{Nd}$. Hence, a possible proximity effect due to the paramagnetic NGO film adjacent to the conducting interface is not apparent from the Hall data discussed here. Notably, the occurrence of the AHE is relatively weak for LGO/STO grown at low deposition pressure ( $p_{\text {dep }}=4 \times 10^{-5}$ mbar, see Fig. 11$)$, while it is more significant for the $\mathrm{LAO} / \mathrm{STO}$ sample grown at higher oxygen pressure $\left(p_{\text {dep }}=1 \times 10^{-3}\right.$ mbar $)$. Hence, the observed trend of an increasingly significant AHE at higher deposition pressure is rather independent of the actual polar material deposited on STO.

The increase of the AHE contribution coincides with the increase in defect density as indicated by the decrease in electron mobility (Fig. 10). As argued above (Fig. 7), this may indicate an increased concentration of cation vacancies in the samples showing the most pronounced AHE. Hence, we suggest that also the AHE and thus the magnetic properties observed here may be related to cationic disorder. Being an intrinsic property of (n-type) STO, the formation of $\mathrm{Sr}$ vacancies is independent of the particular material composition of the heterostructure consistent with the observation of the AHE in the various material systems. However, magnetism in STO is typically related to $\mathrm{Ti}^{3+}$ states. Hence, as acceptor-type defects, $\mathrm{V}_{\mathrm{Sr}}^{\prime \prime}$ should rather reduce the $\mathrm{Ti}^{3+}$ content at the interface (as observed for the high-mobility channel). Therefore, one would rather expect that such defects have a negative effect on magnetism. In fact, a direct link between cation vacancies and magnetic moment or exchange coupling is not intuitive, and details on how cation vacancies may affect magnetism require further verification. Here, we note that this link does not necessarily imply that the cation vacancies themselves directly mediate magnetic ordering. However, there may be an indirect correlation between defects and magnetism, e.g., via the increased scattering rate further localizing the electrons at increased defect density or via mechanical strain induced by vacancies. The latter scenario was addressed in a recent report suggesting that strontium vacancies cause a tetragonal distortion of the STO unit cell, which induces magnetism and an associated Kondolike resistance upturn in $n$-type STO [64]. The altered magnetic properties of the NGO/STO interface we observe in this study may thus be related to a structural distortion, e.g., ionic displacements and buckling or lattice spacing $[19,84-86]$, changing with the cationic defect configuration and growth parameters.

\section{CONCLUSIONS}

We discuss the electronic and magnetic properties of NGO/STO heterointerfaces as well as their ionic defect structure by analyzing the low-temperature transport properties. At temperatures $\lesssim 30 \mathrm{~K}$, Hall measurements reveal the typical nonlinear field dependence of the Hall resistance frequently observed in complex oxide heterostructures. This behavior is found consistent with two-carrier transport attributed to contributions of high-mobility and lowmobility electrons. For $T \lesssim 10 \mathrm{~K}$, we find an additional anomalous Hall effect contribution indicating the presence of coupled magnetic moments forming below a critical temperature.

We introduce a general model describing the entire complex $B$-field behavior of the Hall effect in magnetic electron systems exhibiting an inherently nonlinear conventional Hall effect. Based on this model, the magnetic properties of oxide interface 2DEGs can be extracted directly from Hall experiments. Moreover, this modeling can be easily adopted for other complex electronic systems beyond oxide interfaces. 
For the electronic system at NGO/STO interfaces, the AHE contribution systematically becomes more and more significant with increasing growth pressure $p_{\text {dep }}$, as indicated by the increased critical temperature $T_{c}$, increased critical field $B_{c}$, and increased saturation resistance $R_{0}^{\mathrm{AHE}}$. All of these parameters are tuned by the growth pressure. In particular, the coupling energy varies on the order of 0.4-1 meV depending on growth pressure, showing that the magnetic moments are weakly coupled and indicating a rather fragile magnetic system.

The observed scalability of the AHE perhaps makes an electronic origin of the interfacial magnetism unlikely. In contrast, it indicates a relation of magnetism and intrinsic defect structure. However, the observed trend with $p_{\text {dep }}$ intuitively contradicts magnetic moments induced via oxygen vacancies. Based on the conventional transport parameters, $\mu_{i}$ and $n_{i}$, we find an increasing defect density with increasing $p_{\text {dep }}$. This trend again rules out a significant effect caused by oxygen vacancies, cation intermixing, antisite defects, or a varied carrier distribution. Instead, the observed behavior makes cation vacancies the most likely relevant defects at the interface.

Based on thermodynamics, defect formation through the Schottky equilibrium is, hence, one important process defining the defect structure at complex oxide interfaces during and after growth. As a quantitative estimate of the sheet density of strontium vacancies, we obtain values between $1 \times 10^{12}$ and $5 \times 10^{12} \mathrm{~cm}^{-2}$ depending on the particular growth pressure. The simultaneous observations of (1) the systematic variation of the AHE contribution with $p_{\text {dep }}$ and (2) a systematic variation of the ionic defect structure with $p_{\text {dep }}$ suggests a correlation between ionic defect structure and magnetism which is stabilized in the most defective samples.

Our results show that even at the level of parts per million, crystal defects can have significant impact on electron transport and magnetism at complex oxide interfaces. In particular, this study indicates the key impact of cationic defects and vacancies, acting as scatter centers and electron traps as well as a source of mechanical strain, on the low-temperature transport of oxide interfaces. The presence of $\mathrm{Sr}$ vacancies is a thermodynamical requirement in STO. Therefore, these defects are present in any (hightemperature-grown) STO-based heterostructure. The choice of suitable growth (and annealing) conditions is a crucial tool to control their concentration as well as the resulting physical effects.

\section{ACKNOWLEDGMENTS}

Work at Stanford/SLAC was supported by the Department of Energy, Office of Basic Energy Sciences, Division of Materials Sciences and Engineering, under Contract No. DE-AC02-76SF00515; FAME, one of six centers of STARnet, a Semiconductor Research Corporation program sponsored by MARCO and DARPA (B. K.); the Gordon and Betty Moore Foundation's EPiQS Initiative through Grant No. GBMF4415 (A. G. S.); and ONR-MURI number N00014-12-1-0976 (M. M.). The W2/W3 program of the Helmholtz association is gratefully acknowledged.

[1] A. Ohtomo and H. Y. Hwang, A High-Mobility Electron Gas at the $\mathrm{LaAlO}_{3} / \mathrm{SrTiO}_{3}$ Heterointerface, Nature (London) 427, 423 (2004).

[2] H. Y. Hwang, Y. Iwasa, M. Kawasaki, B. Keimer, N. Nagaosa, and Y. Tokura, Emergent Phenomena at Oxide Interfaces, Nat. Mater. 11, 103 (2012).

[3] D. G. Schlom and J. Mannhart, Oxide ElectronicsInterface Takes Charge over Si, Nat. Mater. 10, 168 (2011).

[4] N. Reyren, S. Thiel, A. D. Caviglia, L. F. Kourkoutis, G. Hammerl, C. Richter, C. W. Schneider, T. Kopp, A.-S. Ruetschi, D. Jaccard, M. Gabay, D. A. Muller, J. M. Triscone, and J. Mannhart, Superconducting Interfaces between Insulating Oxides, Science 317, 1196 (2007).

[5] A. Brinkman, M. Huijben, M. V. Zalk, J. Huijben, U. Zeitler, J. C. Maan, W. G. V. der Wiel, G. Rijnders, D. H. A. Blank, and H. Hilgenkamp, Magnetic Effects at the Interface between Non-Magnetic Oxides, Nat. Mater. 6, 493 (2007).

[6] L. Li, C. Richter, J. Mannhart, and R. C. Ashoori, Coexistence of Magnetic Order and Two-Dimensional Superconductivity at $\mathrm{LaAlO}_{3} / \mathrm{SrTiO}_{3}$ Interfaces, Nat. Phys. 7, 762 (2011).

[7] J. A. Bert, B. Kalisky, C. Bell, M. Kim, Y. Hikita, H. Y. Hwang, and K. A. Moler, Direct Imaging of the Coexistence of Ferromagnetism and Superconductivity at the $\mathrm{LaAlO}_{3} / \mathrm{SrTiO}_{3}$ Interface, Nat. Phys. 7, 767 (2011).

[8] Ariando, X. Wang, G. Baskaran, Z. Q. Liu, J. Huijben, J. B. Yi, A. Annadi, A. R. Barman, A. Rusydi, S. Dhar, Y. P. Feng, J. Ding, H. Hilgenkamp, and T. Venkatesan, Electronic Phase Separation at the $\mathrm{LaAlO}_{3} / \mathrm{SrTiO}_{3}$ Interface, Nat. Commun. 2, 188 (2011).

[9] J. S. Lee, Y. W. Xie, H. K. Sato, C. Bell, Y. Hikita, H. Y. Hwang, and C. C. Kao, Titanium $d_{x y}$ Ferromagnetism at the $\mathrm{LaAlO}_{3} / \mathrm{SrTiO}_{3}$ Interface, Nat. Mater. 12, 703 (2013).

[10] D. Stornaiuolo, C. Cantoni, G. M. D. Luca, R. D. Capua, E. D. Gennaro, G. Ghiringhelli, B. Jouault, D. Marre, D. Massarotti, F. M. Granozio, I. Pallecchi, C. Piamonteze, S. Rusponi, F. Tafuri, and M. Salluzzo, Tunable Spin Polarization and Superconductivity in Engineered Oxide Interfaces, Nat. Mater. 15, 278 (2016).

[11] F. Baiutti, G. Logvenov, G. Gregori, G. Cristiani, Y. Wang, W. Sigle, P. A. van Aken, and J. Maier, High-Temperature Superconductivity in Space-Charge Regions of Lanthanum Cuprate Induced by Two-Dimensional Doping, Nat. Commun. 6, 8586 (2015).

[12] R. A. De Souza, F. Gunkel, S. Hoffmann-Eifert, and R. Dittmann, Finite-Size versus Interface-Proximity Effects in Thin-Film Epitaxial $\mathrm{SrTiO}_{3}$, Phys. Rev. B 89, 241401 (2014).

[13] F. Gunkel, R. Waser, A. H. H. Ramadan, R. A. De Souza, S. Hoffmann-Eifert, and R. Dittmann, Space Charges and Defect Concentration Profiles at Complex Oxide Interfaces, Phys. Rev. B 93, 245431 (2016). 
[14] G. Berner, M. Sing, H. Fujiwara, A. Yasui, Y. Saitoh, A. Yamasaki, Y. Nishitani, A. Sekiyama, N. Pavlenko, T. Kopp, C. Richter, J. Mannhart, S. Suga, and R. Claessen, Direct $k$-Space Mapping of the Electronic Structure in an Oxide-Oxide Interface, Phys. Rev. Lett. 110, 247601 (2013).

[15] A. Joshua, J. Ruhman, S. Pecker, E. Altman, and S. Ilani, Gate-Tunable Polarized Phase of Two-Dimensional Electrons at the $\mathrm{LaAlO}_{3} / \mathrm{SrTiO}_{3}$ Interface, Proc. Natl. Acad. Sci. U.S.A. 110, 9633 (2013).

[16] A. Joshua, S. Pecker, J. Ruhman, E. Altman, and S. Ilani, A Universal Critical Density Underlying the Physics of Electrons at the $\mathrm{LaAlO}_{3} / \mathrm{SrTiO}_{3}$ Interface, Nat. Commun. 3, 1129 (2012).

[17] G. Khalsa and A. H. MacDonald, Theory of the $\mathrm{SrTiO}_{3}$ Surface State Two-Dimensional Electron Gas, Phys. Rev. B 86, 125121 (2012).

[18] F. Gunkel, S. Hoffmann-Eifert, R. Dittmann, S. Mi, C. Jia, P. Meuffels, and R. Waser, High Temperature Conductance Characteristics of $\mathrm{LaAlO}_{3} / \mathrm{SrTiO}_{3}$-Heterostructures under Equilibrium Oxygen Atmospheres, Appl. Phys. Lett. 97, 012103 (2010).

[19] A. Kalabukhov, Y. A. Boikov, I. T. Serenkov, V. I. Sakharov, J. Brjesson, N. Ljustina, E. Olsson, D. Winkler, and T. Claeson, Improved Cationic Stoichiometry and Insulating Behavior at the Interface of $\mathrm{LaAlO}_{3} / \mathrm{SrTiO}_{3}$ Formed at High Oxygen Pressure during Pulsed-Laser Deposition, Europhys. Lett. 93, 37001 (2011).

[20] F. Gunkel, P. Brinks, S. Hoffmann-Eifert, R. Dittmann, M. Huijben, J. E. Kleibeuker, G. Koster, G. Rijnders, and R. Waser, Influence of Charge Compensation Mechanisms on the Sheet Electron Density at Conducting $\mathrm{LaAlO}_{3} / \mathrm{SrTiO}_{3}$-Interfaces, Appl. Phys. Lett. 100, 052103 (2012).

[21] F. Gunkel, S. Wicklein, S. Hoffmann-Eifert, P. Meuffels, P. Brinks, M. Huijben, G. Rijnders, R. Waser, and R. Dittmann, Transport Limits in Defect-Engineered $\mathrm{LaAlO}_{3} /$ $\mathrm{SrTiO}_{3}$ Bilayers, Nanoscale 7, 1013 (2015).

[22] E. Flekser, M. Ben Shalom, M. Kim, C. Bell, Y. Hikita, H. Y. Hwang, and Y. Dagan, Magnetotransport Effects in Polar versus Non-Polar $\mathrm{SrTiO}_{3}$ Based Heterostructures, Phys. Rev. B 86, 121104 (2012).

[23] M. Ben Shalom, C. W. Tai, Y. Lereah, M. Sachs, E. Levy, D. Rakhmilevitch, A. Palevski, and Y. Dagan, Anisotropic Magnetotransport at the $\mathrm{SrTiO}_{3} / \mathrm{LaAlO}_{3}$ Interface, Phys. Rev. B 80, 140403 (2009).

[24] C. Xu, C. Bäumer, R. A. Heinen, S. Hoffmann-Eifert, F. Gunkel, and R. Dittmann, Disentanglement of Growth Dynamic and Thermodynamic Effects in $\mathrm{LaAlO}_{3} / \mathrm{SrTiO}_{3}$ Heterostructures, Sci. Rep. 6, 22410 (2016).

[25] F. Bi, M. Huang, S. Ryu, H. Lee, C.-W. Bark, C.-B. Eom, P. Irvin, and J. Levy, Room-Temperature ElectronicallyControlled Ferromagnetism at the $\mathrm{LaAlO}_{3} / \mathrm{SrTiO}_{3}$ Interface, Nat. Commun. 5, 5019 (2014).

[26] M. Salluzzo, S. Gariglio, D. Stornaiuolo, V. Sessi, S. Rusponi, C. Piamonteze, G. M. De Luca, M. Minola, D. Marré, A. Gadaleta, H. Brune, F. Nolting, N. B. Brookes, and G. Ghiringhelli, Origin of Interface Magnetism in $\mathrm{BiMnO}_{3} / \mathrm{SrTiO}_{3}$ and $\mathrm{LaAlO}_{3} / \mathrm{SrTiO}_{3}$ Heterostructures, Phys. Rev. Lett. 111, 087204 (2013).
[27] M. R. Fitzsimmons, N. W. Hengartner, S. Singh, M. Zhernenkov, F. Y. Bruno, J. Santamaria, A. Brinkman, M. Huijben, H. J. A. Molegraaf, J. de la Venta, and I. K. Schuller, Upper Limit to Magnetism in $\mathrm{LaAlO}_{3} / \mathrm{SrTiO}_{3}$ Heterostructures, Phys. Rev. Lett. 107, 217201 (2011).

[28] R. Pentcheva and A. E. Pickett, Electronic Phenomena at Complex Oxide Interfaces: Insights from First Principles, J. Phys. Condens. Matter 22, 043001 (2010).

[29] R. Pentcheva and W. E. Pickett, Charge Localization or Itineracy at $\mathrm{LaAlO}_{3} / \mathrm{SrTiO}_{3}$ Interfaces: Hole Polarons, Oxygen Vacancies, and Mobile Electrons, Phys. Rev. B 74, 035112 (2006).

[30] N. Pavlenko, T. Kopp, E. Y. Tsymbal, G. A. Sawatzky, and J. Mannhart, Magnetic and Superconducting Phases at the $\mathrm{LaAlO}_{3} / \mathrm{SrTiO}_{3}$ Interface: The Role of Interfacial Ti $3 d$ Electrons, Phys. Rev. B 85, 020407 (2012).

[31] J. Ruhman, A. Joshua, S. Ilani, and E. Altman, Competition between Kondo Screening and Magnetism at the $\mathrm{LaAlO}_{3} / \mathrm{SrTiO}_{3}$ Interface, Phys. Rev. B 90, 125123 (2014).

[32] F. Gunkel, K. Skaja, A. Shkabko, R. Dittmann, S. Hoffmann-Eifert, and R. Waser, Stoichiometry Dependence and Thermal Stability of Conducting $\mathrm{NdGaO}_{3} / \mathrm{SrTiO}_{3}$ Heterointerfaces, Appl. Phys. Lett. 102, 071601 (2013).

[33] A. Annadi, A. Putra, Z. Q. Liu, X. Wang, K. Gopinadhan, Z. Huang, S. Dhar, T. Venkatesan, and Ariando, Electronic Correlation and Strain Effects at the Interfaces between Polar and Nonpolar Complex Oxides, Phys. Rev. B 86, 085450 (2012).

[34] U. Treske, N. Heming, M. Knupfer, B. Buechner, A. Koitzsch, E. Di Gennaro, U. Scotti di Uccio, F. Miletto Granozio, and S. Krause, Observation of Strontium Segregation in $\mathrm{LaAlO}_{3} / \mathrm{SrTiO}_{3}$ and $\mathrm{NdGaO}_{3} / \mathrm{SrTiO}_{3}$ Oxide Heterostructures by X-Ray Photoemission Spectroscopy, APL Mater. 2, 012108 (2014).

[35] F. Luis, M. D. Kuz'min, F. Bartolomé, V. M. Orera, J. Bartolomé, M. Artigas, and J. Rubín, Magnetic Susceptibility of $\mathrm{NdGaO}_{3}$ at Low Temperatures: A Quasi-TwoDimensional Ising Behavior, Phys. Rev. B 58, 798 (1998).

[36] N. Nagaosa, J. Sinova, S. Onoda, A. H. MacDonald, and N. P. Ong, Anomalous Hall Effect, Rev. Mod. Phys. 82, 1539 (2010).

[37] S. Seri and L. Klein, Antisymmetric Magnetoresistance of the $\mathrm{SrTiO}_{3} / \mathrm{LaAlO}_{3}$ Interface, Phys. Rev. B 80, 180410 (2009).

[38] E. M. Pugh, Hall Effect and the Magnetic Properties of Some Ferromagnetic Materials, Phys. Rev. 36, 1503 (1930).

[39] E. M. Pugh and T. W. Lippert, Hall e.m.f. and Intensity of Magnetization, Phys. Rev. 42, 709 (1932).

[40] J. Kötzler and W. Gil, Anomalous Hall Resistivity of Cobalt Films: Evidence for the Intrinsic Spin-Orbit Effect, Phys. Rev. B 72, 060412 (2005).

[41] A. Fete, C. Cancellieri, D. Li, D. Stornaiuolo, A. D. Caviglia, S. Gariglio, and J.-M. Triscone, Growth-Induced Electron Mobility Enhancement at the $\mathrm{LaAlO}_{3} / \mathrm{SrTiO}_{3}$ Interface, Appl. Phys. Lett. 106, 051604 (2015).

[42] J. S. Kim, S. S. A. Seo, M. F. Chisholm, R. K. Kremer, H.-U. Habermeier, B. Keimer, and H. N. Lee, Nonlinear Hall Effect and Multichannel Conduction in $\mathrm{LaTiO}_{3} / \mathrm{SrTiO}_{3}$ Superlattices, Phys. Rev. B 82, 201407 (2010). 
[43] P. Gallagher, M. Lee, T. A. Petach, S. W. Stanwyck, J. R. Williams, K. Watanabe, T. Taniguchi, and D. GoldhaberGordon, A High-Mobility Electronic System at an Electrolyte-Gated Oxide Surface, Nat. Commun. 6, 6437 (2015).

[44] D. J. Keeble, S. Wicklein, R. Dittmann, L. Ravelli, R. A. Mackie, and W. Egger, Identification of $A$ - and B-Site Cation Vacancy Defects in Perovskite Oxide Thin Films, Phys. Rev. Lett. 105, 226102 (2010).

[45] S. Wicklein, A. Sambri, S. Amoruso, X. Wang, R. Bruzzese, A. Koehl, and R. Dittmann, Pulsed Laser Ablation of Complex Oxides: The Role of Congruent Ablation and Preferential Scattering for the Film Stoichiometry, Appl. Phys. Lett. 101, 131601 (2012).

[46] C. Xu, S. Wicklein, A. Sambri, S. Amoruso, M. Moors, and R. Dittmann, Impact of the Interplay between Nonstoichiometry and Kinetic Energy of the Plume Species on the Growth Mode of $\mathrm{SrTiO}_{3}$ Thin Films, J. Phys. D 47, 034009 (2014).

[47] J. H. Song, T. Susaki, and H. Y. Hwang, Enhanced Thermodynamic Stability of Epitaxial Oxide Thin Films, Adv. Mater. 20, 2528 (2008).

[48] L. F. Kourkoutis, J. H. Song, H. Y. Hwang, and D. A. Muller, Microscopic Origins for Stabilizing RoomTemperature Ferromagnetism in Ultrathin Manganite Layers, Proc. Natl. Acad. Sci. U.S.A. 107, 11682 (2010).

[49] A. Sambri, D. Christensen, F. Trier, Y. Chen, S. Amoruso, N. Pryds, R. Bruzzese, and X. Wang, Plasma Plume Effects on the Conductivity of Amorphous $-\mathrm{LaAlO}_{3} / \mathrm{SrTiO}_{3}$ Interfaces Grown by Pulsed Laser Deposition in $\mathrm{O}_{2}$ and $\mathrm{Ar}$, Appl. Phys. Lett. 100, 231605 (2012).

[50] C. Aruta, S. Amoruso, R. Bruzzese, X. Wang, D. Maccariello, F. Miletto Granozio, and U. Scotti di Uccio, Pulsed Laser Deposition of $\mathrm{SrTiO}_{3} / \mathrm{LaGaO}_{3}$ and $\mathrm{SrTiO}_{3} / \mathrm{LaAlO}_{3}$ : Plasma Plume Effects, Appl. Phys. Lett. 97, 252105 (2010).

[51] T. Ohnishi, M. Lippmaa, T. Yamamoto, S. Meguro, and H. Koinuma, Improved Stoichiometry and Misfit Control in Perovskite Thin Film Formation at a Critical Fluence by Pulsed Laser Deposition, Appl. Phys. Lett. 87, 241919 (2005).

[52] A. Kalabukhov, Y. A. Boikov, I. T. Serenkov, V. I. Sakharov, T. Claeson, and D. Winkler, Cation Stoichiometry and Electrical Transport Properties of the $\mathrm{NdGaO}_{3} /(001) \mathrm{SrTiO}_{3}$ Interface, J. Phys. Condens. Matter 27, 255004 (2015).

[53] R. Moos and K. H. Haerdtl, Defect Chemistry of DonorDoped and Undoped Strontium Titanate Ceramics between $1000^{\circ}$ and $1400^{\circ} \mathrm{C}$, J. Am. Ceram. Soc. 80, 2549 (1997).

[54] Y. Kozuka, Y. Hikita, C. Bell, and H. Y. Hwang, Dramatic Mobility Enhancements in Doped $\mathrm{SrTiO}_{3}$ Thin Films by Defect Management, Appl. Phys. Lett. 97, 012107 (2010).

[55] D. M. Smyth, The Defect Chemistry of Metal Oxides (Oxford University Press, New York, 2000).

[56] R. Meyer, R. Waser, J. Helmbold, and G. Borchardt, Observation of Vacancy Defect Migration in the Cation Sublattice of Complex Oxides by ${ }^{18} \mathrm{O}$ Tracer Experiments, Phys. Rev. Lett. 90, 105901 (2003).

[57] B. Rahmati, J. Fleig, W. Sigle, E. Bischoff, J. Maier, and M. Rhle, Oxidation of Reduced Polycrystalline Nb-Doped $\mathrm{SrTiO}_{3}$ : Characterization of Surface Islands, Surf. Sci. 595, 115 (2005).
[58] J. Crawford and P. Jacobs, Point Defect Energies for Strontium Titanate: A Pair-Potentials Study, J. Solid State Chem. 144, 423 (1999).

[59] R. Meyer, R. Waser, J. Helmbold, and G. Borchardt, Cationic Surface Segregation in Donor-Doped $\mathrm{SrTiO}_{3}$ under Oxidizing Conditions, J. Electroceram. 9, 101 (2002).

[60] K. Gomann, G. Borchardt, M. Schulz, A. Gomann, W. Maus-Friedrichs, B. Lesage, O. Kaitasov, S. HoffmannEifert, and T. Schneller, Sr Diffusion in Undoped and La-Doped $\mathrm{SrTiO}_{3}$ Single Crystals under Oxidizing Conditions, Phys. Chem. Chem. Phys. 7, 2053 (2005).

[61] W. Menesklou, H. Schreiner, K. H. Haerdtl, and E. Ivers-Tiffee, High Temperature Oxygen Sensors Based on Doped $\mathrm{SrTiO}_{3}$, Sens. Actuators B 59, 184 (1999).

[62] K. Szot, W. Speier, U. Breuer, R. Meyer, J. Szade, and R. Waser, Formation of Micro-Crystals on the (100) Surface of $\mathrm{SrTiO}_{3}$ at Elevated Temperatures, Surf. Sci. 460, 112 (2000).

[63] M. Akthar, Z. Akthar, R. Jackson, and C. Catlow, ComputerSimulation Studies of Strontium-Titanate, J. Am. Ceram. Soc. 78, 421 (1995).

[64] A. Sarantopoulos, E. Ferreiro-Vila, V. Pardo, C. Magén, M. H. Aguirre, and F. Rivadulla, Electronic Degeneracy and Intrinsic Magnetic Properties of Epitaxial $\mathrm{Nb}: \mathrm{SrTiO}_{3}$ Thin Films Controlled by Defects, Phys. Rev. Lett. 115, 166801 (2015).

[65] C. Bell, S. Harashima, Y. Kozuka, M. Kim, B. G. Kim, Y. Hikita, and H. Y. Hwang, Dominant Mobility Modulation by the Electric Field Effect at the $\mathrm{LaAlO}_{3} / \mathrm{SrTiO}_{3}$ Interface, Phys. Rev. Lett. 103, 226802 (2009).

[66] B. Kalisky, J. A. Bert, C. Bell, Y. Xie, H. K. Sato, M. Hosoda, Y. Hikita, H. Y. Hwang, and K. A. Moler, Scanning Probe Manipulation of Magnetism at the $\mathrm{LaAlO}_{3} / \mathrm{SrTiO}_{3}$ Heterointerface, Nano Lett. 12, 4055 (2012).

[67] Y. Lyanda-Geller, S. H. Chun, M. B. Salamon, P. M. Goldbart, P. D. Han, Y. Tomioka, A. Asamitsu, and Y. Tokura, Charge Transport in Manganites: Hopping Conduction, the Anomalous Hall Effect, and Universal Scaling, Phys. Rev. B 63, 184426 (2001).

[68] H. C. Yang, L. M. Wang, and H. E. Horng, Anomalous Hall Effect of $\mathrm{Nd}_{0.7} \mathrm{Sr}_{0.3} \mathrm{MnO}_{3}$ Films with Large Magnetoresistance Ratio: Evidence of Berry Phase Effect, Phys. Rev. B 64, 174415 (2001).

[69] E. Lesne, N. Reyren, D. Doennig, R. Mattana, H. Jaffres, V. Cros, F. Petroff, F. Choueikani, P. Ohresser, R. Pentcheva, A. Barthelemy, and M. Bibes, Suppression of the Critical Thickness Threshold for Conductivity at the $\mathrm{LaAlO}_{3} / \mathrm{SrTiO}_{3}$ Interface, Nat. Commun. 5, 4291 (2014).

[70] R. Waser, Bulk Conductivity and Defect Chemistry of Acceptor-Doped Strontium Titanate in the Quenched State, J. Am. Ceram. Soc. 74, 1934 (1991).

[71] R. Pentcheva, M. Huijben, K. Otte, W. E. Pickett, J. E. Kleibeuker, J. Huijben, H. Boschker, D. Kockmann, W. Siemons, G. Koster, H. J. W. Zandvliet, G. Rijnders, D. H. A. Blank, H. Hilgenkamp, and A. Brinkman, Parallel Electron-Hole Bilayer Conductivity from Electronic Interface Reconstruction, Phys. Rev. Lett. 104, 166804 (2010).

[72] M. Huijben, G. Rijnders, D. H. A. Blank, S. Bals, S. V. Aert, J. Verbeeck, G. V. Tendeloo, A. Brinkman, and H. Hilgenkamp, Electronically Coupled Complementary 
Interfaces between Perovskite Band Insulators, Nat. Mater. 5, 556 (2006).

[73] C. Cancellieri, N. Reyren, S. Gariglio, A. D. Caviglia, A. Fete, and J. Triscone, Influence of the Growth Conditions on the $\mathrm{LaAlO}_{3} / \mathrm{SrTiO}_{3}$ Interface Electronic Properties, Europhys. Lett. 91, 17004 (2010).

[74] A. D. Caviglia, S. Gariglio, C. Cancellieri, B. Sacepe, A. Fete, N. Reyren, M. Gabay, A. F. Morpurgo, and J.-M. Triscone, Two-Dimensional Quantum Oscillations of the Conductance at $\mathrm{LaAlO}_{3} / \mathrm{SrTiO}_{3}$ Interfaces, Phys. Rev. Lett. 105, 236802 (2010).

[75] A. McCollam, S. Wenderich, M. K. Kruize, V. K. Guduru, H. J. A. Molegraaf, M. Huijben, G. Koster, D. H. A. Blank, G. Rijnders, A. Brinkman, H. Hilgenkamp, U. Zeitler, and J. C. Maan, Quantum Oscillations and Subband Properties of the Two-Dimensional Electron Gas at the $\mathrm{LaAlO}_{3} / \mathrm{SrTiO}_{3}$ Interface, APL Mater. 2, 022102 (2014).

[76] M. Minohara, Y. Hikita, C. Bell, H. Inoue, M. Hosoda, H. K. Sato, H. Kumigashira, M. Oshima, E. Ikenaga, and H. Y. Hwang, The Potential Profile at the $\mathrm{LaAlO}_{3} / \mathrm{SrTiO}_{3}$ (001) Heterointerface in Operando Conditions, arXiv:1403.5594.

[77] S. Gariglio, A. Fete, and J.-M. Triscone, Electron Confinement at the $\mathrm{LaAlO}_{3} / \mathrm{SrTiO}_{3}$ Interface, J. Phys. Condens. Matter 27, 283201 (2015).

[78] L. Yu and A. Zunger, A Polarity-Induced Defect Mechanism for Conductivity and Magnetism at Polar-Nonpolar Oxide Interfaces, Nat. Commun. 5, 5118 (2014).

[79] T. D. Sanders, M. T. Gray, F. J. Wong, and Y. Suzuki, $\mathrm{LaAlO}_{3} / \mathrm{SrTiO}_{3}$ Interfaces Doped with Rare-Earth Ions, Phys. Rev. B 91, 205112 (2015).

[80] M. L. Reinle-Schmitt, C. Cancellieri, A. Cavallaro, G. F. Harrington, S. J. Leake, E. Pomjakushina, J. A. Kilner, and P. R. Willmott, Chemistry and Structure of Homoepitaxial
$\mathrm{SrTiO}_{3}$ Films and Their Influence on Oxide-Heterostructure Interfaces, Nanoscale 6, 2598 (2014).

[81] M. P. Warusawithana, C. Richter, J. A. Mundy, P. Roy, J. Ludwig, S. Paetel, T. Heeg, A. A. Pawlicki, L. F. Kourkoutis, M. Zheng, M. Lee, B. Mulcahy, W. Zander, Y. Zhu, J. Schubert, J. N. Eckstein, D. A. Muller, C. S. Hellberg, J. Mannhart, and D. G. Schlom, $\mathrm{LaAlO}_{3}$ Stoichiometry Is Key to Electron Liquid Formation at $\mathrm{LaAlO}_{3} / \mathrm{SrTiO}_{3}$ Interfaces, Nat. Commun. 4, 2351 (2013).

[82] H. K. Sato, C. Bell, Y. Hikita, and H. Y. Hwang, Stoichiometry Control of the Electronic Properties of the $\mathrm{LaAlO}_{3} / \mathrm{SrTiO}_{3}$ Heterointerface, Appl. Phys. Lett. 102, 251602 (2013).

[83] A. H. Reshak, M. Piasecki, S. Auluck, I. V. Kityk, R. Khenata, B. Andriyevsky, C. Cobet, N. Esser, A. Majchrowski, M. Wirkowicz, R. Diduszko, and W. Szyrski, Effect of $U$ on the Electronic Properties of Neodymium Gallate $\left(\mathrm{NdGaO}_{3}\right)$ : Theoretical and Experimental Studies, J. Phys. Chem. B 113, 15237 (2009).

[84] C. L. Jia, S. B. Mi, M. Faley, U. Poppe, J. Schubert, and K. Urban, Oxygen Octahedron Reconstruction in the $\mathrm{SrTiO}_{3} / \mathrm{LaAlO}_{3}$ Heterointerfaces Investigated Using Aberration-Corrected Ultrahigh-Resolution Transmission Electron Microscopy, Phys. Rev. B 79, 081405 (2009).

[85] P. R. Willmott, S. A. Pauli, R. Herger, C. M. Schlepütz, D. Martoccia, B. D. Patterson, B. Delley, R. Clarke, D. Kumah, C. Cionca, and Y. Yacoby, Structural Basis for the Conducting Interface between $\mathrm{LaAlO}_{3}$ and $\mathrm{SrTiO}_{3}$, Phys. Rev. Lett. 99, 155502 (2007).

[86] R. Yamamoto, C. Bell, Y. Hikita, H. Y. Hwang, H. Nakamura, T. Kimura, and Y. Wakabayashi, Structural Comparison ofn-Type and p-Type $\mathrm{LaAlO}_{3} / \mathrm{SrTiO}_{3}$ Interfaces, Phys. Rev. Lett. 107, 036104 (2011). 